\title{
Optical characterization of CdSe nanocrystals
}

\author{
Cheng-yuan Chen ${ }^{\mathrm{a}}$, Jia-Ren Lee ${ }^{\mathrm{a}}$, Chi-Ta Chia ${ }^{\mathrm{a}}$, Chien-Rong Lu ${ }^{\mathrm{a}, *}$, \\ I-Shuo Liu ${ }^{\mathrm{b}}$, Wei-Fang $\mathrm{Su}^{\mathrm{b}}$ \\ ${ }^{a}$ Department of Physics, National Taiwan Normal University, Taipei 116, Taiwan, ROC \\ ${ }^{\mathrm{b}}$ Institute of Material Science and Engineering, National Taiwan University, Taipei 116, Taiwan, ROC
}

\begin{abstract}
We have investigated the optical properties of CdSe nanocrystals by using the transmittance and photoluminescence spectroscopy. The samples used in the optical experiment are nanocrystals of cylindrical rods and spheres dispersed in toluene. The transition features in the photoluminescence spectra agree with the transition edges observed in the transmittance spectra. Theoretical models based on the effective mass approximation with infinite barriers were established for both types of nanocrystals. The calculated transition energies and oscillator strengths are compared with the observed spectral features. Improved models with the finite barrier and shallow potential step to account for the capping contribute to minor corrections of the order of thousandths.
\end{abstract}

(C) 2007 Elsevier Ltd. All rights reserved.

Keywords: A. Semiconductor; A. Nanostructure; D. Optical properties

\section{Introduction}

The size of nanocrystals is of the order of several to tens nanometers which is close to the de Broglie wavelength of the electron and hole. Physically, nanocrystal is one of the low-dimensional semiconductor systems called quantum dot. In the quantum wire or quantum well systems, there are one or two translational degrees of freedom and a large number of free electrons and holes due to the large number of density of states. In the quantum dot system, there is no translational symmetry, and all degrees of freedom are confined. Nanocryatals have atomic like electronic configurations, photon-induced phenomena and very different optical properties from the bulk crystals. From the atomic or molecular points of view, nanocrystals can be regarded as large molecules. They are usually dispersed uniformly in the transparent solid or liquid for characterization. Based on the special optical properties of quantum dots and nanocrystals, many applications have been developed [1-6]. The optical properties such as line shape analysis have not been well understood yet. In this report, we investigated the absorption spectra of $\mathrm{CdSe}$ rods and spheres. The

\footnotetext{
*Corresponding author. Fax: + 886229326408.

E-mail address: lupond@phy03.phys.ntnu.edu.tw (C.-R. Lu).
}

transition energies and oscillator strengths are calculated by the effective mass approximation models and can be used as the basis for line shape analysis in the future.

\section{Experimental}

The CdSe nanocrystal was synthesized using the $\mathrm{CdO}$ one-pot approach [7,8]. The reaction mixture of cadmium oxide (CdO), tetradecylphosphonic acid (TDPA), and trioctylphosphine oxide (TOPO) is heated up to $300^{\circ} \mathrm{C}$ in argon, and turned into homogeneous solution. After introducing seleniumin and trioctylphosohine (TOP) solution, and reacting for several minutes, slowly cooled down to room temperature. The brown CdSe powder obtained after methanol rinsing could be dispersed in toluene and hexane. Transmission electron microscopy (TEM) pictures in Fig. 1 show the rod shape of the TDPA- and TOPOcapped CdSe nanocrystals.

CdSe nanocrystals were dispersed in toluene for transmission measurements. A $150 \mathrm{~W}$ xenon arc lamp was used as light source. The wavelength was selected by a quartermeter monochromator. The probe light is detected by a Si detector after transmitted through the sample and was normalized by the bare, toluene only, light intensity. In the photoluminescence (PL) experiment, the excitation 

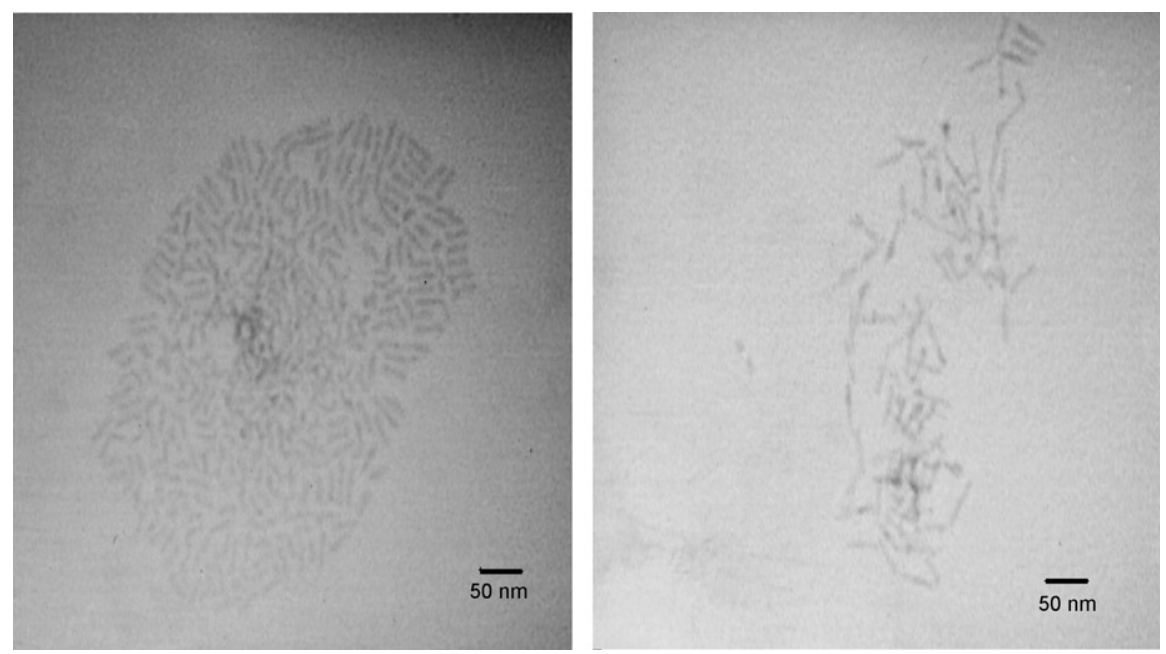

Fig. 1. TEM pictures of the $5 \times 15 \mathrm{~nm}$ and $5 \times 25 \mathrm{~nm}$ nanorods.

photons were provided by the $476 \mathrm{~nm}$ line of an argon-ion laser.

\section{Results and discussion}

Fig. 2 presents the absorption spectra from the transmittance spectroscopy of CdSe nanorods. The absorption edges are blue shifted as the size of the nanocrystal is reduced. The PL spectra of CdSe nanocrystals are also shown in Fig. 2. The transition features in the PL spectra agree with those observed in the absorption. Optical transition features blue shifted as the size of the nanocrystal becomes smaller due to quantum-confinement effect.

For the nanorod, we start with a rigid cylinder effective mass model with infinite potential barriers. The wave functions have cylindrical symmetry; the radial component is a Bessel function while the $z$ component is a sinusoidal function as follows:

$\varphi_{q s t}=\sqrt{\frac{2}{\pi b\left[J_{m}^{\prime}\left(K_{m n} a\right)\right]}} J_{m}\left(K_{m n} \rho\right) \sin \left(k_{q} z\right) e^{\mathrm{i} m \phi}$,

where $q, m$, and $n$ are quantum numbers, $J_{m}\left(K_{m} \rho\right)$ is the Bessel function, and $J_{m}^{\prime}\left(K_{m n} a\right)$ is the first derivative of the Bessel function at radius $a$. Solving the rigid boundary condition equations would generate the allowed wave vectors $k_{q}=n_{q} \pi / b$ and $K_{m n}=y_{m n} / a$, where $y_{m n}$ are roots of Bessel function $J_{m}\left(K_{m n} a\right)=0$. Eigenenergies may be obtained from the energy-momentum relation:

$E_{q m n}=\frac{\hbar^{2}}{2 M}\left(K_{m n}^{2}+k_{q}^{2}\right)=\frac{\hbar^{2}}{2 M}\left[\left(\frac{y_{m n}}{a}\right)^{2}+\left(\frac{n_{q} \pi}{b}\right)^{2}\right]$.

The transition energy will be the sum of the bulk gap energy, electron subband energy, and hole subband energy. The bulk band gap energy and the effective masses are taken from the literature [9]. Fig. 3 shows the variation of transition energies versus rod length for different radii. The transition energy is more sensitive to the radius than to the

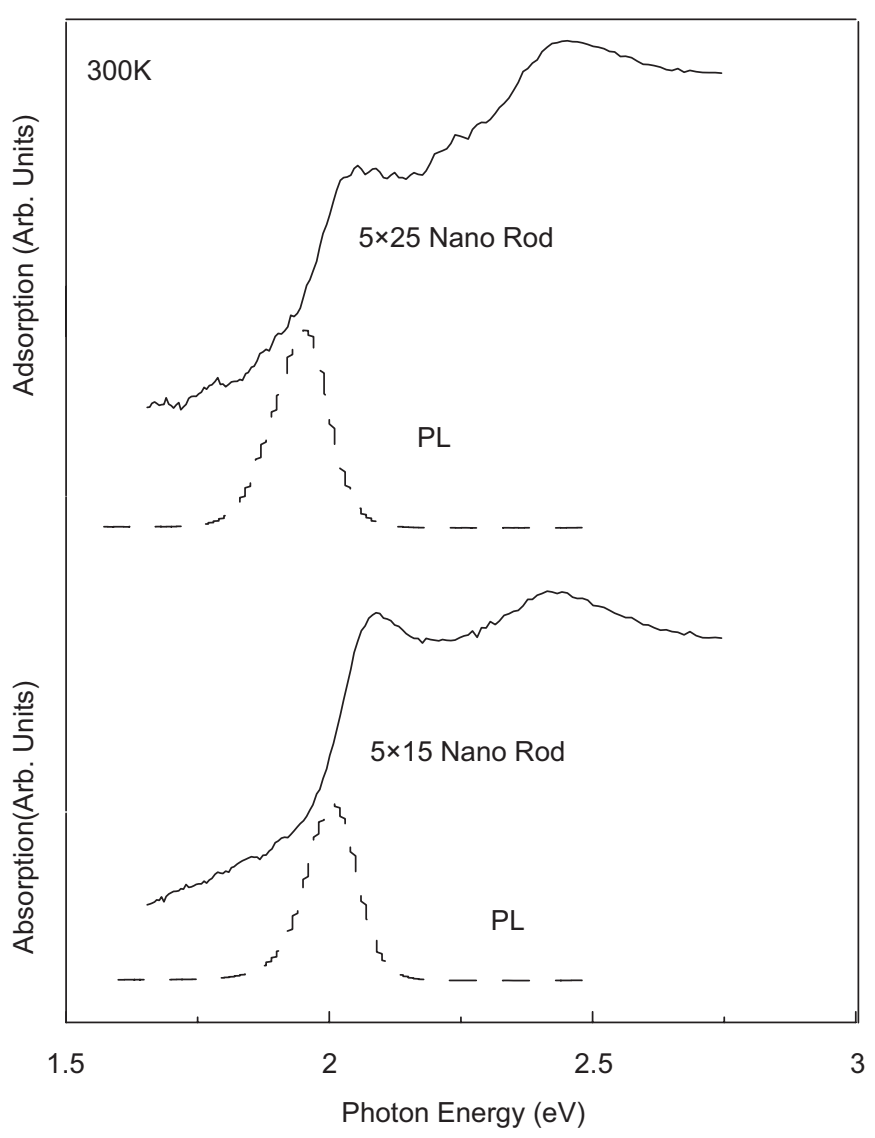

Fig. 2. Absorption and PL spectra of CdSe nanorods.

length. The calculated major transition energies are compared with the observed spectra in Fig. 4. Vertical bars indicate the position of theoretically calculated transition energies, and their lengths represent the oscillator strengths $f \equiv\left(2 m_{0} \omega / 3 \hbar\right)\left|\left\langle\phi_{m}|r| \phi_{\ell}\right\rangle\right|^{2}$ obtained by integrating the dipole matrix element. 


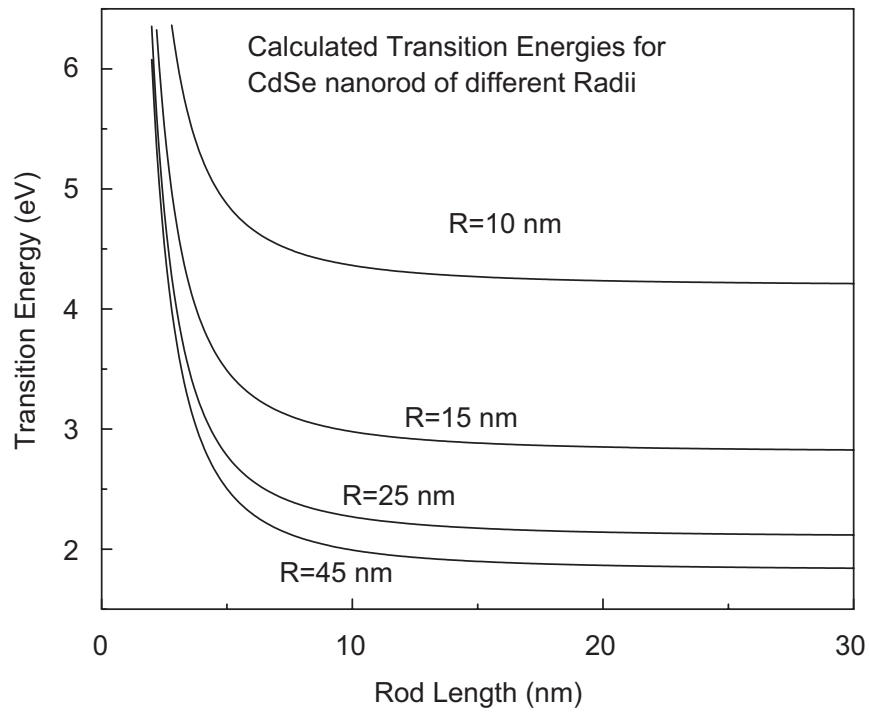

Fig. 3. Variation of transition energies of the CdSe nanorods versus the length and radius.

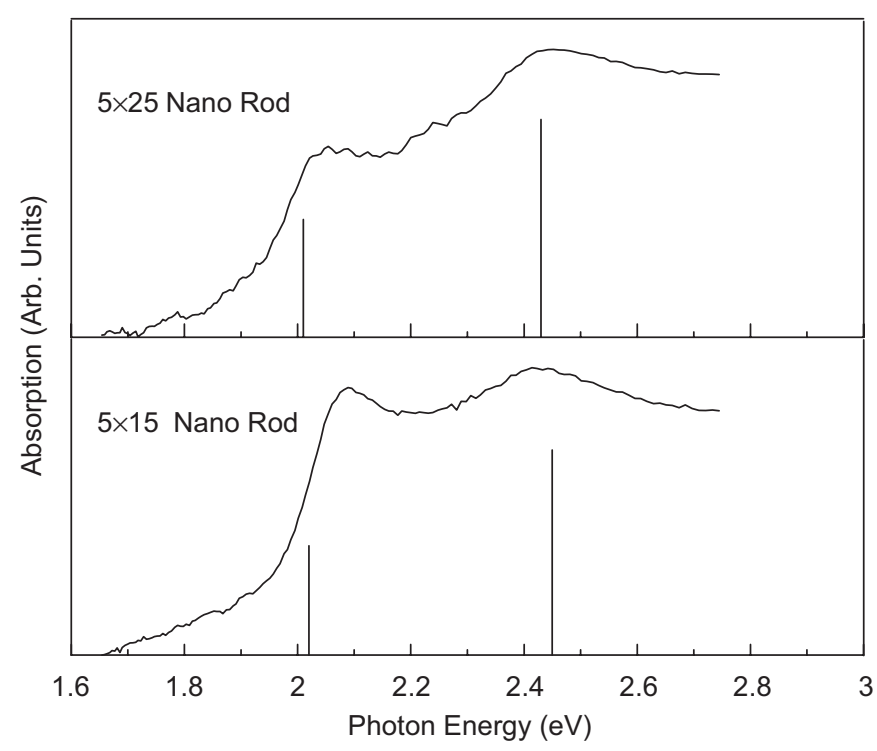

Fig. 4. Calculated transition energies and oscillator strengths compared with the observed spectra for nanorods.

As to the nanospheres, a rigid sphere effective mass model will have wave functions with spherical symmetry as

$\varphi_{n l m}=\sqrt{\frac{2}{a^{3}\left[j_{l}^{\prime}\left(k_{l n} a\right)\right]^{2}}} j_{l}\left(k_{l n} \rho\right) Y_{l}^{m}(\theta, \phi)$,

where $n, l$, and $m$ are quantum numbers, $Y_{l}^{m}(\theta, \phi)$ and $j_{l}\left(k_{l n} \rho\right)$ are spherical harmonic and spherical Bessel functions. Solving rigid sphere boundary condition equation, $j_{l}\left(k_{\ln } a\right)=0$, would generate allowed wave vectors and eigenenergies. With the bulk band gap energy included, the radius dependence of the transition energies may be obtained as shown in Fig. 5. The calculated major

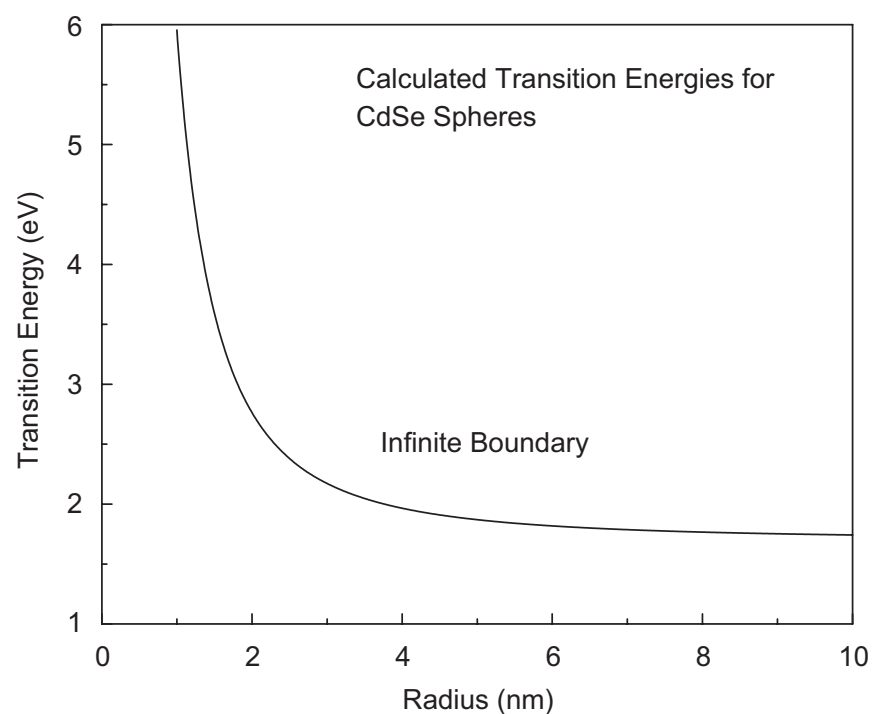

Fig. 5. Variation of transition energies of the CdSe nanospheres versus radius.

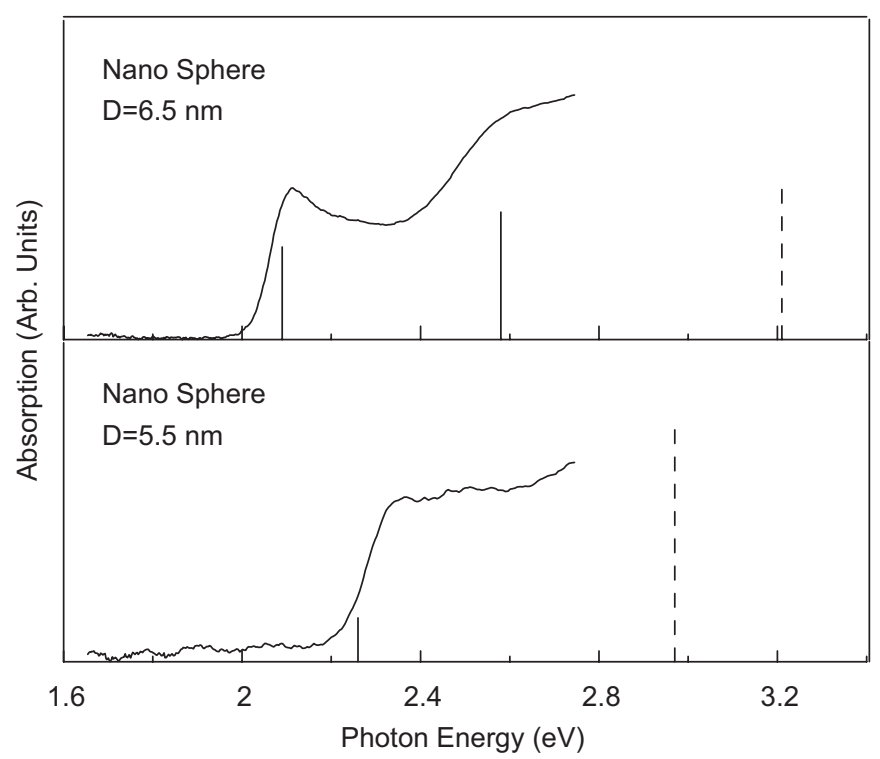

Fig. 6. Calculated transition energies and oscillator strengths compared with the observed spectra for nanospheres.

transition energies of nanospheres are compared with observed spectra in Fig. 6. Only those with transition probabilities comparably large are indicated by vertical bars. The lengths of vertical bars are proportional to the calculated oscillator strength. Solid bars indicate the observed transitions while dashed bars indicate those beyond the spectroscopic range.

Two improved models have been used to describe the nanocrystals better. Instead of suspending in vacuum, the investigated nanocrystals are capped with TDPA and TOPO and dispersed in the solvent. Wave functions could penetrate through the boundary and have finite values outside the nanocrystals, while the rigid model of infinite 
barriers requires zero wave function at the boundary. With finite barriers of several electron volts, the calculated transition energy is about several thousandths less than those of infinite barriers. The other improvement is inserting a potential step to represent the TDPA and TOPO capping. Since the coating is thin and the band offset is about $1.2 \mathrm{eV}$, the correction of the transition energies is also about several thousandths of those of infinite barriers.

\section{Summary}

The optical properties of nanorods and nanospheres made of CdSe with different sizes were investigated by transmittance spectroscopy. The PL spectroscopy was also used to study the recombination transition energy. The rigid models of infinite barrier potentials with the effective mass approximation have been established for both nanorods and nanospheres. The calculated transition energies and oscillator strengths are comparable to the spectroscopy results. Improved models such as finite barriers and a thin shallow step to describe the capping contribute to corrections with the order of thousandths of those calculated from the rigid models.

\section{Acknowledgments}

Financial support from National Science Council (NSC 92-2112-M-003-014) and National Taiwan Normal University is gratefully acknowledged.

\section{References}

[1] M. Bruchez Jr., M. Moronne, P. Gin, S. Weiss, A.P. Alivisators, Science 281 (1988) 2013.

[2] W.C.W. Chan, S. Nie, Science 281 (1998) 2016.

[3] V. Colvin, M. Schlamp, A.P. Alivisators, Nature 370 (1994) 354.

[4] B.O. Dabbousi, M.G. Bawendi, O. Onotsuka, M.F. Rubner, Appl. Phys. Lett. 66 (1995) 1316.

[5] W.C. Chan, S. Nei, Science 281 (1998) 2016.

[6] V.I. Klimov, et al., Science 290 (2000) 314.

[7] Z.A. Peng, X. Peng, Am. Chem. Soc. 123 (2001) 1389-1395.

[8] X. Peng, Adv. Mater. 15 (2003) 459.

[9] H.J. LozykowS-Ky, V.K. Shastri, J. Appl. Phys. 69 (1991) 3235. 